\title{
Long term variations in the Extreme UV corona: the EIT/SoHO perspective
}

\author{
J-F. Hochedez, F. Clette, E. Verwichte, D. Berghmans, P. Cugnon \\ Royal Observatory of Belgium, Solar Physics Department, Circular \\ Avenue 3., B-1180 Brussels Belgium, hochedez@oma.be, sol.oma.be
}

\begin{abstract}
Since the start of the SoHO mission, EIT -the Extreme ultraviolet Imaging Telescope- offers a global view of the solar corona over the whole rising phase of the current activity cycle. Such a dataset is unprecedented. We give here the current results of an on-going investigation over the entire EIT archive. In the Fe XV images ( $2 \mathrm{MK}$ ), the on-disk and off-disk intensity distributions have been evaluated, and their evolution is described. Additionally, we developed an image processing technique that extracts the smallest detectable features. The cosmic ray hits are statistically disentangled from the solar point-like phenomena, and the trends in both rates are assessed.
\end{abstract}

\section{Introduction}

Among SoHO coronal experiments, EIT, the Extreme-ultraviolet Imaging Telescope (Delaboudinière et al. 1995; Moses et al. 1997) provides global highresolution views of the low corona. Scanning the EIT archive -systematically and automatically - will pave the way to investigations in different fields of solar physics. Comparable synoptic surveys have been undertaken with success by other space and ground-based imager teams : e.g. SXT and HXT of Yohkoh, LASCO and MDI of SoHO, and naturally for all dedicated instruments providing extended time series. The EIT collection (Table 1) amounts to a total of

\begin{tabular}{lrr}
\hline Wavelength & Number of images FF/FR & Number of images FF/HR \\
\hline $17.1 \mathrm{~nm}$ Fe IX/X & 5581 & 2479 \\
$19.5 \mathrm{~nm}$ Fe XII & 61858 & 38437 \\
$28.4 \mathrm{~nm} \mathrm{Fe} \mathrm{XV}$ & 5596 & 1415 \\
$30.4 \mathrm{~nm}$ He II & 9563 & 3307 \\
\hline \hline
\end{tabular}

Table 1. Status of the EIT archive as of September 2000. The full field (FF) is 45 arcmin wide, full resolution (FR) gives $1024 \times 1024$ pixels, half resolution (HR) gives $512 \times 512$ pixels.

approximately 170000 images. It is realistic to assume that EIT observations will cover a full cycle until some successor prolongs the monitoring. However, almost five years of daily scrutiny have already passed, and the time is ripe to tackle the archive as a whole. We have first focussed on the Fe XV bandpass as it allows dealing with smaller, more regular data series. 


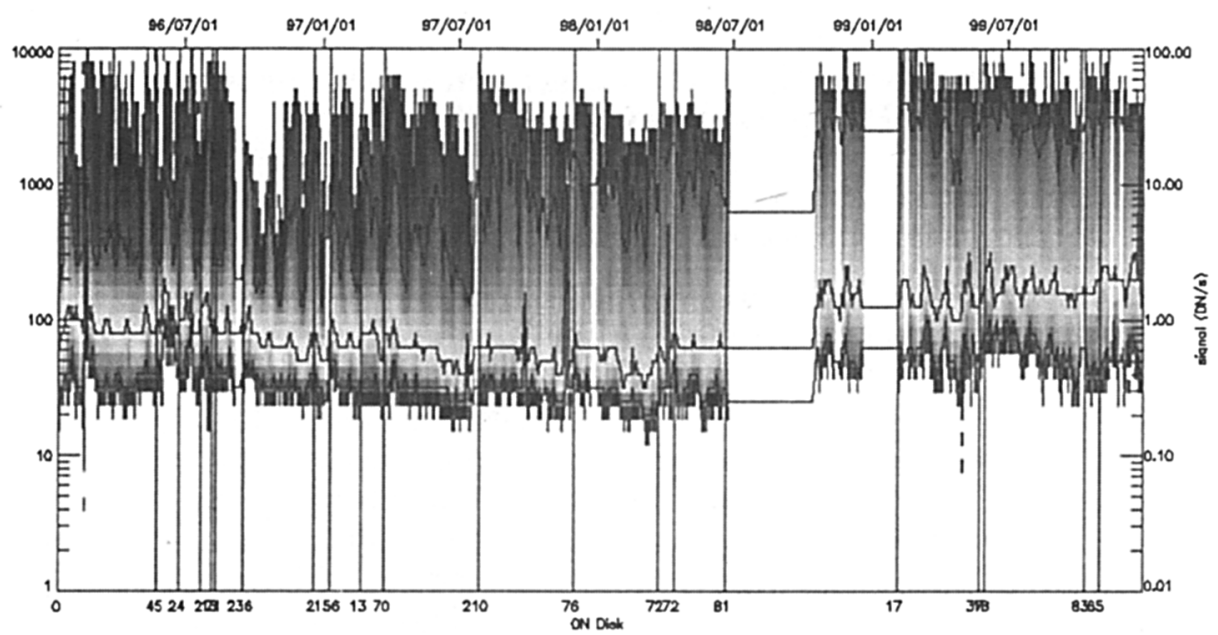

Figure 1. The on-disk intensity distributions of EIT Fe XV images from January 1996 to December 1999. Each vertical slice is a median distribution over two days. The vertical lines indicate the bakeout periods with their durations below. The lowest significantly represented, the most represented and the highest significantly represented intensities are plotted. Intensities and occurrences have log scales.

\section{Intensity distribution evolution}

The a priori easiest quantitative parameters to extract from EUV solar images are of photometric nature. It suffers unfortunately from instrumental and operational drawbacks. The main ones owe to the badly estimated loss of sensitivity that is only partly recovered through recurrent bakeouts of the detector. However, the photometric approach is instructive, and it is the required first step toward on-flight calibrations and the desirable radiometric advances. Each analysed full-field $28.4 \mathrm{~nm}$ images went through the following steps : 1/ The image is corrected for the electronic offset, the grid shadow, the pre-flight flatfield, and converted to logarithmic scale. 2/ The image is split into "on-disk" $(<95 \% \mathrm{R} \odot)$, "off-disk" ( $>105 \% \mathrm{R} \odot)$, and "limb" (in-between). As it exhibits singularities, the limb region is excluded from this study. 3/ The intensity histograms are computed on-disk and off-disk. 4/ The histograms of the log intensities were shifted to take into account the variable exposure time, the filter configuration and the binning.

In Figure 1, the histograms are nicely modulated if there are only one or two active regions, during e.g. 1996. Later the picture is more intricate. The signal seems strongly affected by the repeated efforts to recover the sensitivity of the detector. Although the pre-flight photometric calibration of the EIT has been finalized (Dere et al. 2000), it is impossible to infer radiometric quantities as long as the degradations are not corrected for. From further analysis, Hochedez et al. (2000) confirm that the increase in EUV flux with rising activity would 


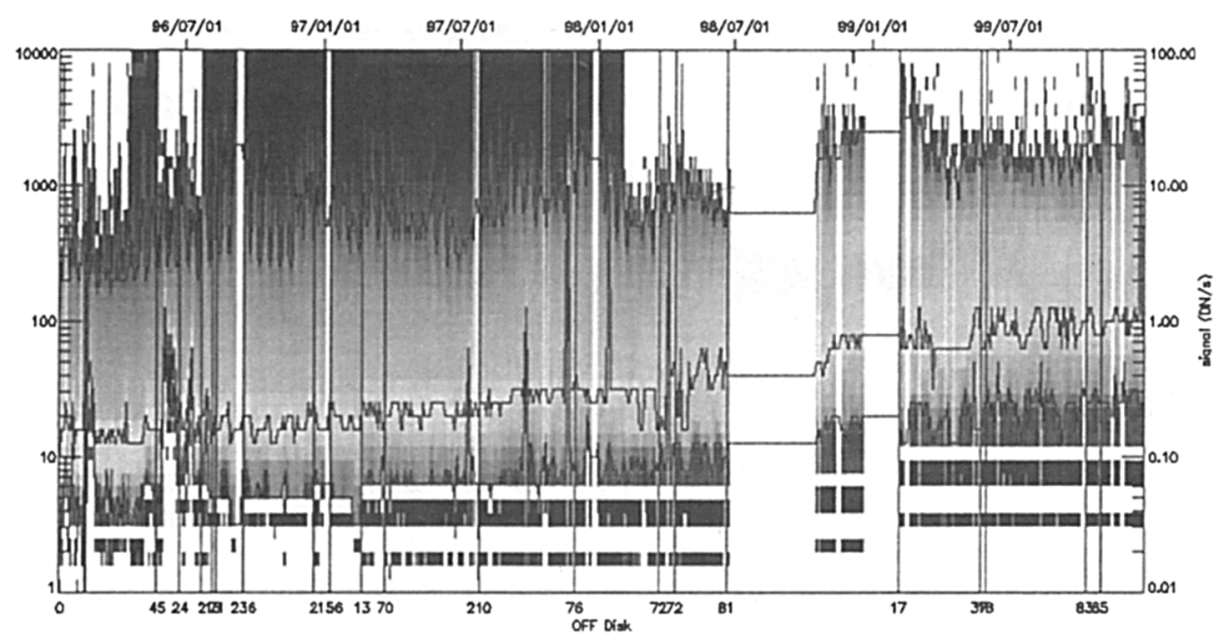

Figure 2. The off-disk intensity distributions of EIT Fe XV images from January 1996 to December 1999. cf. Figure 1 for the context

originate in the expansion of ARs. Their average brightness seems conversely to be fairly constant, and their maximum brightness to be controlled by the filling factors, and by the instrument resolution. Off-disk (Figure 2), the active regions -observed once per solar rotation on-disk- are seen twice at the East and West limbs. The sensitivity could be maintained because the median EUV flux has been smaller. The latter has increased exponentially by a factor 8 from early 1996 to late 1999. Physically, this is interpreted as the cumulative emission of the canopy that develops with the magnetic cycle. In the same interval, the sunspot number increased by 20 , and the $10.7 \mathrm{~cm}$ radio flux by 2.3 .

\section{Density evolution of small solar structures}

A complementary line consists in the use of pattern recognition techniques. Although it pushes the current methods to their limits, it proves feasible to segment EUV solar structures. The principal constraints originate in the high CPU requirements that contradict the purpose of scanning the whole archive, and in the programming standards not compatible with the otherwise prevalent IDL language, expanded by the Solar Soft library. As a first application, we report about the smallest features in EIT Fe XV images. We developed a nonlinear transformation that replaces each pixel by a "best estimate value" built from the $5 \times 5$ neighbourhood. It is named "Minimum Plane Fit" as a plane is fitted through the lowest pixels of the neighbourhood, and the central pixel is interpolated. It proves to efficiently extract the smallest bright objects with a threshold based on the photon statistics. Features are then counted, on-disk or off-disk, as a function of the level of the background on which they occur. Darker off-disk $(>1.05 \mathrm{R} \odot)$ regions cannot exhibit any solar point-like source. This is where the cosmic ray hits rate is estimated to be steady around a value 


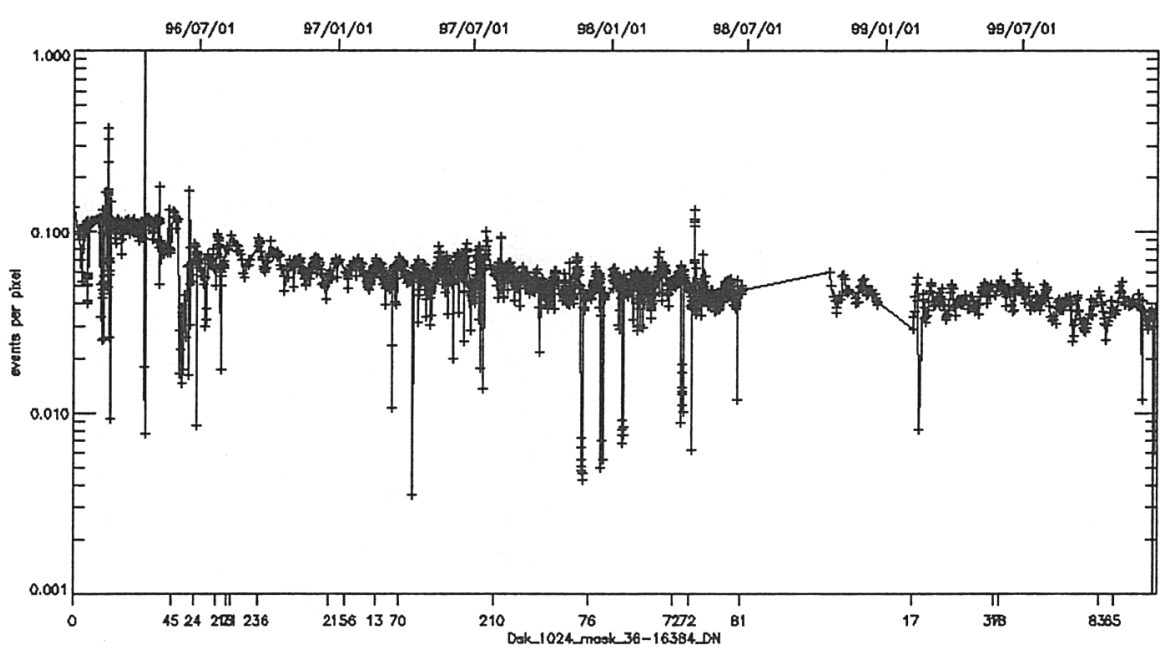

Figure 3. Decrease of the bright points and loop footpoints densities for the brighter regions of the Fe XV solar disk. The excesses come from proton showers or telemetry faults, the troughs issue from absorbing prominences that augment the area but not the bright points rate.

of $4.10^{-5} \mathrm{evt} / \mathrm{pxl} / \mathrm{s}$. In contrast, on-disks densities of small bright features are higher and not much a function of exposure time. The extracted entities that are not cosmic hits appear to be bright points or magnetic loop footpoints. The remarkable result is the regular decrease of the density of solar point-like features from $4 \%$ (of the solar disk area) early 1996 down to $1 \%$ late 1999 in the darker regions (i.e. coronal holes). These tiny features are visually identified as bright points. In the more intense part of the solar disk, the density of small objects is higher since many magnetic loop footpoints are included in the count. Still, there is a decline from $12 \%$ to $3 \%$ (Figure 3 ). These results confirm observations from SXT (Nakakubo \& Hara, 2000), but could possibly be fully or partly attributed to instrumental degradations.

Acknowledgments. The authors acknowledge the support from PRODEX, and from the Belgian government (OSTC). SOHO is a project of international cooperation between ESA and NASA.

\section{References}

Delaboudinière, J-P., et al. 1995, Sol.Phys., 162, 291

Dere, K., et al. 2000, Sol.Phys., 195, 13

Hochedez, J-F., et al. 2000, ESA-SP 463

Moses, D., et al. 1997, Sol.Phys., 175, Issue 2, 571

Nakakubo, K., Hara, H. 2000, Advances in Space Research, 25, Issue 9, 1905 